\title{
Gas Adsorption in Active Carbons and the Slit-Pore Model 1: Pure Gas Adsorption
}

\author{
M. B. Sweatman ${ }^{\dagger}$ and N. Quirke* \\ Department of Chemistry, Imperial College, South Kensington, London, SW7 2AY, UK \\ Received: October 15, 2004; In Final Form: March 22, 2005
}

\begin{abstract}
We describe procedures based on the polydisperse independent ideal slit-pore model, Monte Carlo simulation and density functional theory (a 'slab-DFT') for predicting gas adsorption and adsorption heats in active carbons. A novel feature of this work is the calibration of gas-surface interactions to a high surface area carbon, rather than to a low surface area carbon as in all previous work. Our models are used to predict the adsorption of carbon dioxide, methane, nitrogen, and hydrogen up to 50 bar in several active carbons at a range of near-ambient temperatures based on an analysis of a single $293 \mathrm{~K}$ carbon dioxide adsorption isotherm. The results demonstrate that these models are useful for relatively simple gases at near-critical or supercritical temperatures.
\end{abstract}

\section{Introduction}

The main aim of our research is to create a fast, quantitatively accurate method for predicting gas mixture adsorption in active carbons and other adsorbents. Activated carbons are used for a variety of purposes. Many of them involve the separation of one fluid component from another on an industrial scale. To design a separation process it is useful to have a phase diagram describing how gases are adsorbed by a given material. For pure fluids we need specify only the bulk pressure and temperature, in the absence of hysteresis, so an adsorbed phase diagram is relatively easy to map. But for mixtures it becomes increasingly difficult and time-consuming to map the adsorbed phase diagram as the number of fluid components increases. Methods that allow mixture phase diagrams to be accurately and rapidly predicted are therefore of considerable interest, but are not yet generally available despite considerable progress in understanding adsorption phenomena. ${ }^{1}$

The approach adopted in this work is to use computationally intensive, i.e., molecular simulation, methods to predict pure gas adsorption, and a fast theory that takes the pure adsorption data as input to predict gas mixture adsorption. This paper, part I, deals with the prediction of the adsorption of pure gases up to relatively high pressure over a range of temperatures based on analysis of one "probe" gas adsorption isotherm. Part II, which follows this paper, describes application of the models described in this paper to "fast" gas mixture adsorption prediction, where only pure gas adsorption data are provided as input. The end result is a method by which gas mixture adsorption in active carbons is predicted quickly and accurately on the basis of a single "characterization" measurement, i.e., the probe gas adsorption isotherm.

This work describes Monte Carlo methods and a "slab-DFT" that models gas adsorption in slit-pores and methods, based on the polydisperse independent ideal slit-pore model, for predicting gas adsorption in active carbons. We use these models to predict the adsorption of several gases, namely carbon dioxide, methane, nitrogen, and hydrogen, in several active carbons up to reasonably high pressure (about 50 bar) over a range of near- critical or supercritical temperatures. The results demonstrate that these models are quite accurate for these gases under these conditions. However, we are cautious in advocating these methods for significantly subcritical, strongly polar, or complex molecular gases. Further work beyond what is presented here is needed to establish whether more detailed models are required to describe adsorption of these gases in active carbons.

A great deal of work already exists in the literature on the subject of predicting pure ${ }^{2-16}$ and mixed gas ${ }^{17-19}$ adsorption at a range of temperatures on the basis of a single probe gas adsorption isotherm, using either DFT or MC simulation. However, all this work, which we have reviewed elsewhere, ${ }^{1}$ is, in our opinion, limited because in every case gas-surface interactions are calibrated to low surface area carbons such as graphite, Sterling, ${ }^{20}$ or Vulcan $3 \mathrm{G} .{ }^{20}$ The most significant contribution of this paper, part $\mathrm{I}$, is that it shows that significantly greater accuracy can be obtained if gas-surface interactions are calibrated to a reference high surface area active carbon. The premise here is that the surfaces of active carbons are more similar to each other than to low surface area carbons. We also describe methods for predicting adsorption for a range of temperatures, from which adsorption heats can be predicted. ${ }^{1}$

The remainder of this paper is organized as follows. First, we present our surface and gas models and our MC simulation and DFT methods. Then we present predictions for pure gas adsorption isotherms, including their temperature variation, on the basis of a single pure carbon dioxide adsorption isotherm. Finally, we conclude with a discussion.

\section{Pure Gas Adsorption and the Slit-Pore Model}

To model gas adsorption in active carbons accurately both a molecular model of the surface and a molecular theory of gas adsorption are required. The surface model should be sufficiently complex that it captures behavior relevant to active carbons, for example capillary condensation, but sufficiently simple that calculations can be performed efficiently, particularly if gas mixtures are to be modeled. In this work we employ the polydisperse independent ideal slit-pore model to model the surface of active carbons.

While the precise nature of the pore surface must depend on the precursor and treatment, there is recent evidence from high- 
resolution electron microscopy ${ }^{21,22}$ (HREM) that for nongraphitizing carbons the pore surface is formed from curved fullerene-like fragments with 5-, 6-, and 7-membered rings. A random arrangement of these elements produces a rather tortuous pore space. Whatever the true nature of the surface the polydisperse independent ideal slit-pore model is by far the most successful ${ }^{1}$ model for characterizing active carbons. With this model adsorption in a number of independent ideal slit-pores with a range of pore widths is summed (or integrated for a continuous distribution) to give the total amount adsorbed per gram of material at a particular pressure

$$
N(P)=\int_{0}^{\infty} \mathrm{d} H_{\mathrm{p}} f\left(H_{\mathrm{p}}\right) v\left(H_{\mathrm{p}}, P\right)
$$

Here, $f\left(H_{p}\right)$ is called a pore-size distribution (PSD) where $H_{\mathrm{p}}$ is the physical width of a pore, ${ }^{23} v\left(H_{\mathrm{p}}, P\right)$ is the kernel of local excess isotherms (for comparison with experiment), and the mass of material is measured in a vacuum. In this work the PSD, which represents the incremental pore volume, is calculated by analyzing a single experimental carbon dioxide adsorption isotherm at $293 \mathrm{~K}$. Adsorption isotherms for any gas at any temperature are then easily calculated by using the appropriate kernel with the optimized PSD.

Clearly, the success of this method depends on three crucial factors: (1) the accuracy and universality (in the sense that one PSD is accurate for every gas at every temperature) of the polydisperse independent ideal slit-pore model, (2) the solution method for obtaining the PSD, and (3) the accuracy of the kernel for each gas and temperature. This paper is essentially testing the first factor, if only for the gases and range of temperatures for which we have experimental data to make comparison.

A great deal of discussion in the literature has focused on the second factor. ${ }^{24,25}$ Various schemes have been devised, including simulated annealing ${ }^{14,26}$ and genetic algorithm ${ }^{27}$ optimization methods. Whatever optimization method is employed, since (1) is a Fredholm equation of the first kind ${ }^{28}$ it can have from zero to an infinite number of solutions depending on the form of the experimental isotherm and the kernel. Davies et al. ${ }^{29}$ have discussed practical considerations for obtaining at most one solution from (1) that essentially constrain the PSD and kernel to comply with the information content of the experimental isotherm.

The third factor concerns the accuracy of a particular method for generating equilibrium isotherms for a given Hamiltonian, or molecular model, and the accuracy of the molecular model. Once again, many different methods have been discussed in the literature, ranging from empirical methods based on Polanyi's potential theory ${ }^{30}$ to methods based on statistical mechanics, such as density functional theory (DFT). In this paper we employ grand-canonical Monte Carlo simulation, which provides essentially exact results within statistical error for a given Hamiltonian. Since we believe our gas models to be quite accurate (see the appendix), this work essentially addresses the usefulness of our surface model, i.e., the polydisperse independent slit-pore model of active carbons, the Steele potential that is used to model individual pores and our method for parametrizing this potential. The slit-pore model is described next.

In a slit-pore the external potential, $V_{i a}^{\text {ext }}(z)$, is the sum of contributions from each wall,

$$
V_{i a}^{\mathrm{ext}}(z)=V_{i a}^{\mathrm{w}}(z)+V_{i a}^{\mathrm{w}}\left(H_{p}-z\right)
$$

where subscript $i a$ indicates site $a$ on molecule $i$. We employ the well-known Steele potential ${ }^{31}$ for $V_{i a}^{\mathrm{w}}$

$$
\begin{aligned}
& V_{i a}^{\mathrm{w}}(z)= \\
& 2 \pi \rho_{w} \sigma_{i a w}{ }^{2} \epsilon_{i a w} \Delta_{w}\left(\frac{2}{5}\left(\frac{\sigma_{i a w}}{z}\right)^{10}-\left(\frac{\sigma_{\text {iaw }}}{z}\right)^{4}-\frac{\sigma_{\text {iaw }}{ }^{4}}{3 \Delta_{w}\left(0.61 \Delta_{w}+z\right)^{3}}\right)
\end{aligned}
$$

where $\rho_{\mathrm{w}}$ is the density of atoms in the wall, $\Delta_{\mathrm{w}}$ is the interlayer spacing between graphitic sheets (taken to be $114 \mathrm{~nm}^{-3}$ and $0.335 \mathrm{~nm}$ respectively ${ }^{31,32}$ ) and $\sigma_{\text {iaw }}$ and $\epsilon_{\text {iaw }}$ are the gas - surface interaction length and strength parameters, respectively. This potential models the two-body interaction between LennardJones gas molecule sites and Lennard-Jones wall atom sites. We do not take explicit account of polar gas-surface or higherbody interactions, or the transferability of our gas molecular models from the bulk environment to the pore (see the appendix), but expect that our calibration method for gassurface interactions (see below) will take account of these complications implicitly, provided they are a small contribution to the overall gas - surface interaction. If we use the LorentzBerthelot combining rules

$$
\sigma_{i j}=\left(\sigma_{i i}+\sigma_{j j}\right) / 2 ; \epsilon_{i j}=\sqrt{\epsilon_{i i} \epsilon_{j j}}
$$

then for a given gas molecular model our surface model is fully defined by definition of the wall-wall interaction length and strength parameters, $\sigma_{w w}$ and $\epsilon_{w w}$ respectively. Note, these parameters can be different for each gas. Calibration of these parameters is the focus of this paper, part 1, and is described next.

Calibration of Gas-Surface Interactions. All previous work of this type in the literature has calibrated gas-surface interactions to a low surface area carbon. This work shows that greater accuracy can be obtained by calibrating these interactions to a high surface area carbon (in this work we use an active carbon codenamed PNC).

Our calibration method has two steps. First, we chose the nitrogen-surface interaction to be identical to commonly used parameters for the nitrogen-graphite interaction in the literature, i.e., we choose $\epsilon_{w w} / k_{\mathrm{B}} T=28.0, \sigma_{w w}=0.34 \mathrm{~nm}$, and use the LB rules (eq 4) to obtain cross-parameters for nitrogen-surface interactions. Then the surface-surface interaction strength, $\epsilon_{w w}$, for every other gas is adjusted, and the LB rules are used again to achieve the best fit to experiment (for each pure gas on PNC) based on the carbon dioxide PSD for this material. The gas molecular models used in this work are new and have been optimized to reproduce bulk properties using Monte Carlo simulation (usually saturation properties obtained from Gibbs ensemble simulations). This exercise is detailed in the appendix. The resulting gas model parameters are given in Table 1 . The kernels for these gases at $293 \mathrm{~K}$ are obtained by grand-canonical MC simulation. ${ }^{14,33}$

Note that because the experimental pore volume is calibrated with helium an effective "chemical" pore width for helium will be required if theory and experiment are to be perfectly consistent. ${ }^{34-36}$ The excess adsorption is then calculated as

$$
N(P)=\int_{0}^{\infty} \mathrm{d} H_{\mathrm{p}} f\left(H_{\mathrm{p}}\right)\left(v^{\mathrm{ab}}\left(H_{\mathrm{p}}, P\right)-\frac{\rho_{b}(P)}{\rho_{\mathrm{He}}} v_{\mathrm{He}}^{\mathrm{ab}}\left(H_{\mathrm{p}}\right)\right)
$$

where $v^{\mathrm{ab}}\left(H_{\mathrm{p}}, P\right)$ is the absolute local adsorption and $v_{\mathrm{He}}^{\mathrm{ab}}\left(H_{\mathrm{p}}\right)$ and $\rho_{\mathrm{He}}$ are the absolute local adsorption and bulk density respectively for helium at the calibration temperature and pressure. However, in this work we use a chemical pore width identical to that in reference, ${ }^{23}$ defined as $H_{\mathrm{c}}=H_{\mathrm{p}}-0.285$ 
TABLE 1: Model Parameters Used in MC Simulations ${ }^{a}$

\begin{tabular}{lllll}
\hline parameter & \multicolumn{1}{c}{$\mathrm{N}_{2}$} & \multicolumn{1}{c}{$\mathrm{CH}_{4}$} & \multicolumn{1}{c}{$\mathrm{CO}_{2}$} & \multicolumn{1}{c}{$\mathrm{H}_{2}$} \\
\hline$\sigma_{f f}(\mathrm{~nm})$ & 0.329 & 0.373 & C: 0.275 & 0.27 \\
& & & O: 0.304 & \\
$r_{\mathrm{c}}(\mathrm{nm})$ & 1.5 & 1.492 & 1.5 & 1.35 \\
$\epsilon_{f f} / k_{\mathrm{B}}(\mathrm{K})$ & 36.6 & 151.5 & C: 28.3 & 8.0 \\
& & & O: 84.2 & \\
$l_{x}(\mathrm{~nm})$ & \pm 0.0547 & 0 & C: 0 & \pm 0.0371 \\
& 0 & & O: \pm 0.1149 & \\
$l_{\mathrm{q}}(\mathrm{nm})$ & 0 & 0 & \pm 0.1149 & \pm 0.0371 \\
& \pm 0.0547 & & 0.6512 & -0.98 \\
& 0.928 & 0 & -0.3256 & 0.49 \\
& -0.464 & & 24.0 & 56.0
\end{tabular}

${ }^{a}$ The terms $l_{x}$ and $l_{q}$ are the $x$-displacements of Lennard-Jones and partial charge sites from the molecular Center, while $q$ is the value of the respective partial charge. $\sigma_{f f}, \epsilon_{f f}, \epsilon_{w w}$ and $r_{c}$ are described in the text.

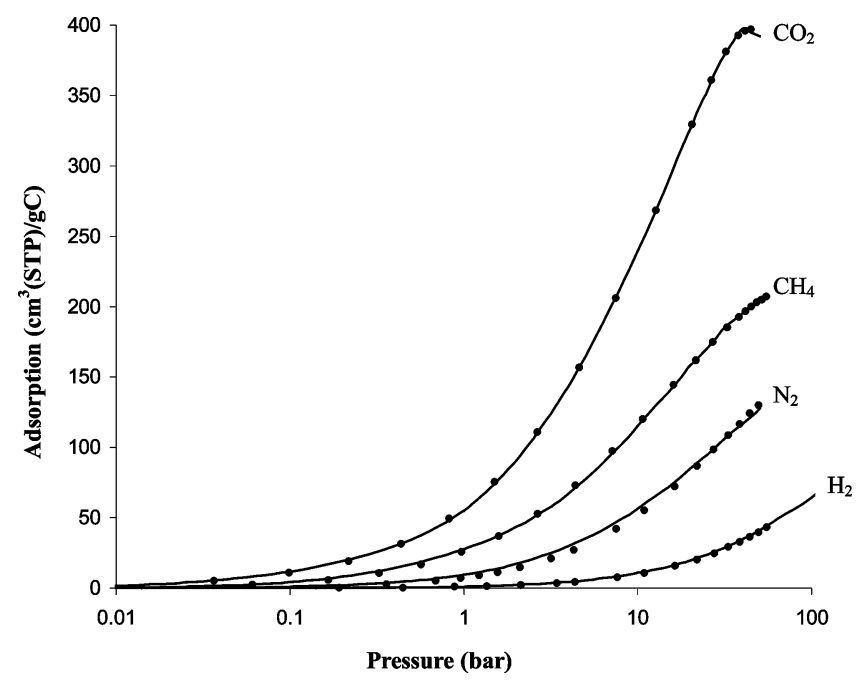

Figure 1. Calibration of gas - surface interaction strength parameters to PNC active carbon. Each line is an isotherm generated by the adsorption integral (eq 1) using the carbon dioxide PSD (see Figure 3) and Monte Carlo simulation kernels. Symbols are experiment. Pressure is on a logarithmic scale.

$\mathrm{nm}$, which leads to inconsistencies with experiment. Nevertheless, we expect that these inconsistencies will be insignificant for the gases, temperatures, and pressures that we employ because our gas - surface interaction calibration method will act to compensate for them. We recognize that this compensatory effect might be inadequate at much higher pressures than those used here.

Figure 1 shows the fitted isotherms for each pure gas: carbon dioxide, methane, nitrogen, and hydrogen on PNC active carbon. The carbon dioxide PSD is obtained using a downhill-simplex ${ }^{28}$ optimization algorithm. It is constrained to a sum of $n$ log-normal modes, ${ }^{14}$ and 10 optimization attempts are made with very different initial conditions. The objective function of each optimization attempt is the root-mean-square deviation (rmsd) of the modeled carbon dioxide isotherm from the experimental data provided it (the rmsd) is greater than the average experimental error. However, if the rmsd is less than the average experimental error, then we optimize a normalized measure of the smoothness (or information content) of the PSD instead; specifically we minimize

$$
\int_{H_{\min }}^{H_{\max }} \mathrm{d} H_{\mathrm{p}}\left|f^{\prime}\left(H_{\mathrm{p}}\right)\right| / \int_{H_{\min }}^{H_{\max }} \mathrm{d} H_{\mathrm{p}} f\left(H_{\mathrm{p}}\right)
$$

We increase the number of modes until at least one of the 10 optimization attempts (with the fixed number of modes) yields a PSD that provides a fit within the average experimental error, starting with just one mode. The resulting PSD is the least sensitive PSD to perturbations, in the experimental data or the kernel, of all possible PSDs that fit the experimental data well (i.e., within experimental error). For almost all active carbons we test this procedure yields a PSD with two modes. We represent $H_{\mathrm{p}}$ on a discrete mesh with $0.1 \mathrm{~nm}$ resolution and convert the integrals in eqs 1,5 , and 6 to summations. We choose $H_{\text {min }}=0$ and let $H_{\max }$ depend on the maximum pressure of the input carbon dioxide isotherm. We have two sets of data provided by different laboratories. One set, which comprises the Vulcan 3G and AX21 results, records pressure up to a maximum of about 15 bar for carbon dioxide isotherms. For analyzing these data we set $H_{\max }$ to $2.5 \mathrm{~nm}$, which corresponds to the pore width beyond which isotherms up to 15 bar are virtually indistinguishable ${ }^{29}$ (in the sense that isotherms in pores wider than this can be almost identically transformed into each other by simply multiplying an entire isotherm by a constant). The other data set measures pressure up to about 50 bar, which corresponds to $H_{\max }$ at $4.5 \mathrm{~nm}$.

Same Temperature Adsorption Prediction. Figure 2 shows predictions for the adsorption of several pure, relatively simple gases at $293 \mathrm{~K}$ on three active carbons up to about 50 bar using the models and methods described above. A PSD is obtained for each carbon from the carbon dioxide isotherm, and this is used to predict the adsorption of the other gases at the same temperature. These are a representative sample of results from nearly 20 different active carbons. In this case we have provided results for three very different carbons, i.e., TA1 is a carbon molecular sieve, TG2 is an "ordinary" active carbon, while AX21 is a super-high surface area carbon. Figure 3 shows the calculated PSDs for each of these carbons. Quite clearly, they are all very different.

Figure 4 shows predictions for Vulcan $3 \mathrm{G}$ using the same approach, i.e., we calculate a PSD for Vulcan $3 \mathrm{G}$ on the basis of the carbon dioxide adsorption isotherm. Vulcan $3 \mathrm{G}$ is a carbon with BET surface area $a^{20,37} \sim 70 \mathrm{~m}^{2} / \mathrm{g}$, which is more than an order of magnitude smaller than typical active carbons. Consequently, we call it a low surface area carbon. It has often $^{14,15,20,38}$ been employed as a reference surface for calibrating gas-surface interactions because it is usually ${ }^{37}$ considered to be nonporous, like Sterling and graphite. In this case a satisfactory fit to the carbon dioxide isotherm can be obtained with a single mode PSD. The relative error in methane and nitrogen adsorption prediction is up to about $20 \%$ for methane and $40 \%$ for nitrogen at the highest pressures, although the absolute error is quite similar for these gases. This indicates that the surface of Vulcan $3 \mathrm{G}$ is quite different to active carbons. Its PSD is also shown in Figure 3. Note that, unlike the active carbons above, Vulcan's PSD does not have significant contributions from pores with widths $<1 \mathrm{~nm}$.

As a further test of the difference between Vulcan $3 \mathrm{G}$ and active carbons we have recalibrated the strength of gas-surface interactions to reproduce adsorption on Vulcan $3 \mathrm{G}$ as follows. We keep the value of the carbon dioxide-surface interaction unchanged, but adjust the methane-surface interaction to provide a good fit for Vulcan $3 \mathrm{G}$. We then calculate a new kernel for methane using this recalibrated potential and make predictions for methane adsorption on active carbon using carbon dioxide PSDs. Note that because we have not changed our carbon dioxide kernel these PSDs are identical to those used in the above results, i.e., those shown in Figure 3. Figure 5 shows the new predictions for methane compared to experiment. 
a)

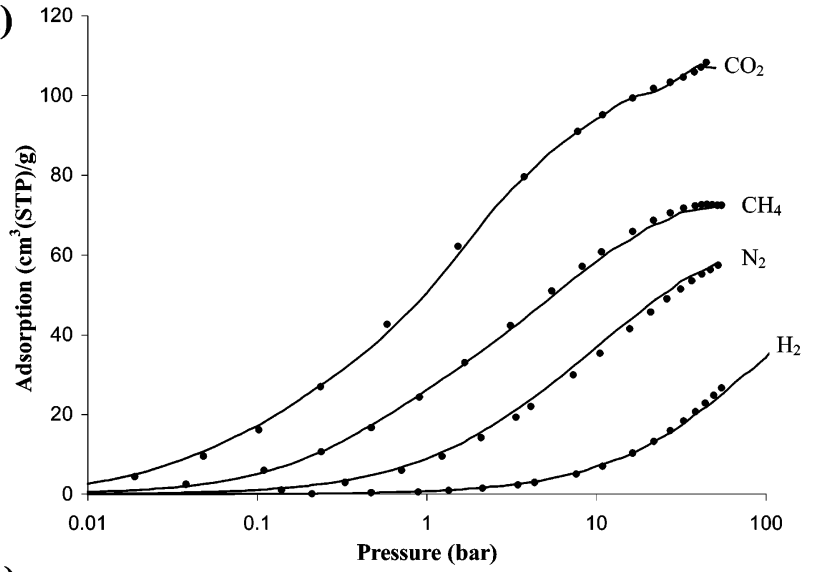

b)

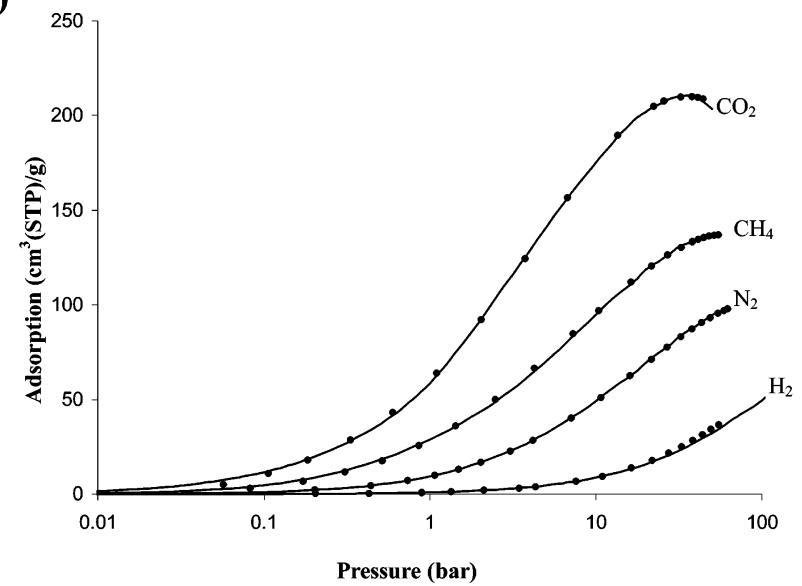

c)

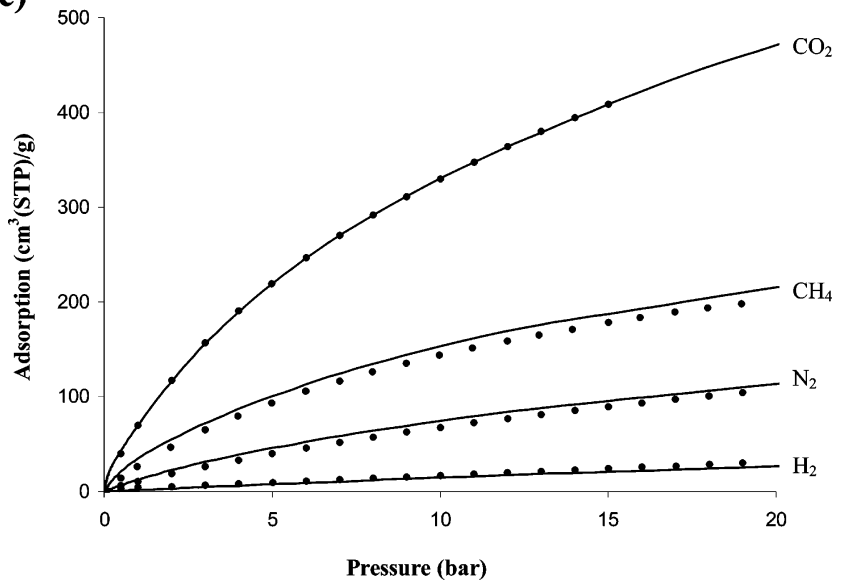

Figure 2. Adsorption isotherms of carbon dioxide, methane, nitrogen, and hydrogen on active carbons: (a) TA1, (b) TG2, and (c) AX21. The methane, nitrogen, and hydrogen isotherms (lines) are predictions based on carbon dioxide slit-PSDs (see Figure 3) and Monte Carlo simulation kernels. Gas-surface interaction strength parameters are calibrated to PNC carbon (see Figure 1). Symbols are experiment. Pressure is on a logarithmic scale for (a) and (b).

It is clear that calibration of gas-surface interactions to Vulcan $3 \mathrm{G}$ leads to poor predictions compared to the earlier results when gas-surface interactions were calibrated to a reference active carbon. Further, we find that it is not possible to obtain completely satisfactory predictions for methane adsorption on Vulcan 3G for any methane-surface interaction strength at all using the carbon dioxide PSD. This suggests there might be an inconsistency in this experimental data.

These results indicate a potential difference between Vulcan 3G (and presumably other low surface area carbons given their similarity ${ }^{37}$ ) and active carbons, other than their surface area.

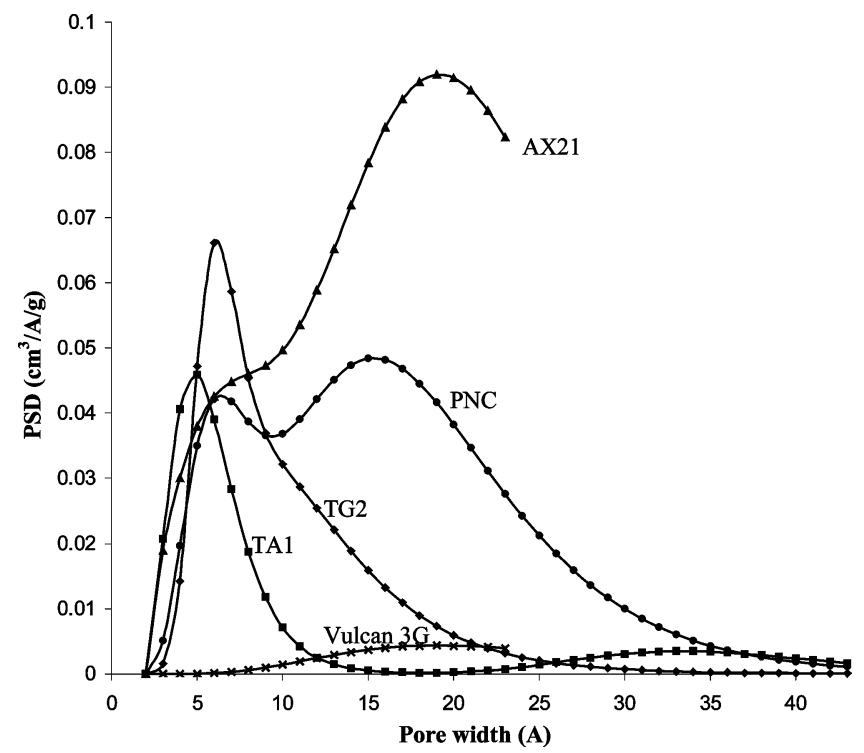

Figure 3. Pore-size distributions (PSDs) for the carbons in Figures 1, 2, 4, and 5; PNC (circles), TA1 (squares), TG2 (diamonds), AX21 (triangles), and Vulcan 3G (diagonal crosses).

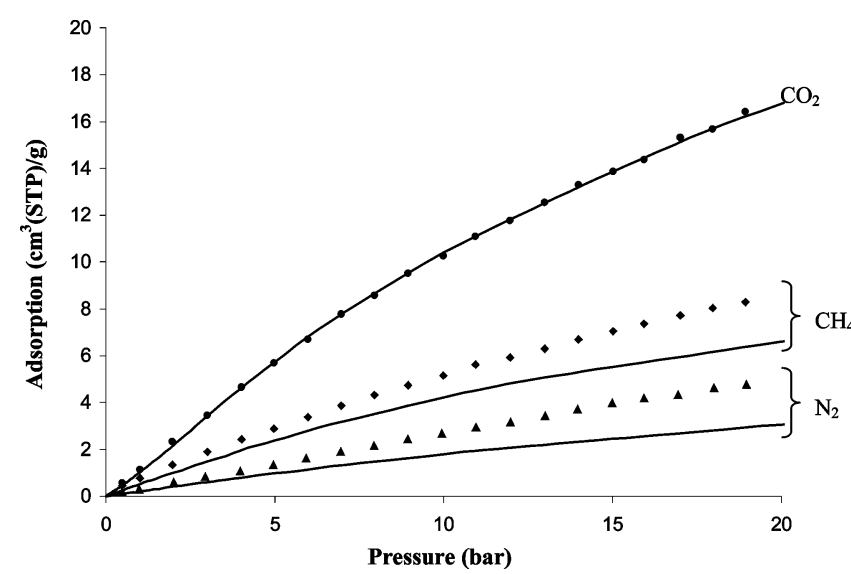

Figure 4. Adsorption isotherms of carbon dioxide (circles), methane (diamonds), and nitrogen (triangles) on Vulcan 3G, a low surface area carbon (see ref 20). The methane and nitrogen predictions (lines) are based on a carbon dioxide slit-PSD (see Figure 3) and Monte Carlo simulation kernels. Gas-surface interaction strength parameters are calibrated to PNC carbon (see Figure 1). Symbols are experiment.

One possible explanation of this difference is that Vulcan 3G is not a significantly porous carbon, so the slit-pore model is inappropriate. However, the adsorption isotherms for Vulcan $3 \mathrm{G}$ in Figure 4 strongly suggest that Vulcan $3 \mathrm{G}$ is significantly heterogeneous, since these isotherms cannot be reproduced by modeling Vulcan $3 \mathrm{G}$ as a simple planar surface ${ }^{14}$ (due to the curvature in the carbon dioxide isotherm). In which case it is not clear how Vulcan 3G should be modeled if it cannot be represented as a planar surface or a combination of slit pores. Alternatively, we suggest that differences in the geometric and chemical construction of surfaces in low and high surface area carbons can lead to quite different "effective" gas-surface interactions (the gas-surface interactions in our models represent contributions from a range of different factors). Of course, we cannot rule out the possibility that these differences are caused by experimental error, given that the Vulcan $3 \mathrm{G}$ and AX21 data were obtained from a different laboratory than the other data, and any systematic error might be exaggerated for Vulcan $3 \mathrm{G}$ relative to the active carbons because of its relatively low uptake. So there would be benefit in repeating this work. Nevertheless, whatever the outcome we should always expect 


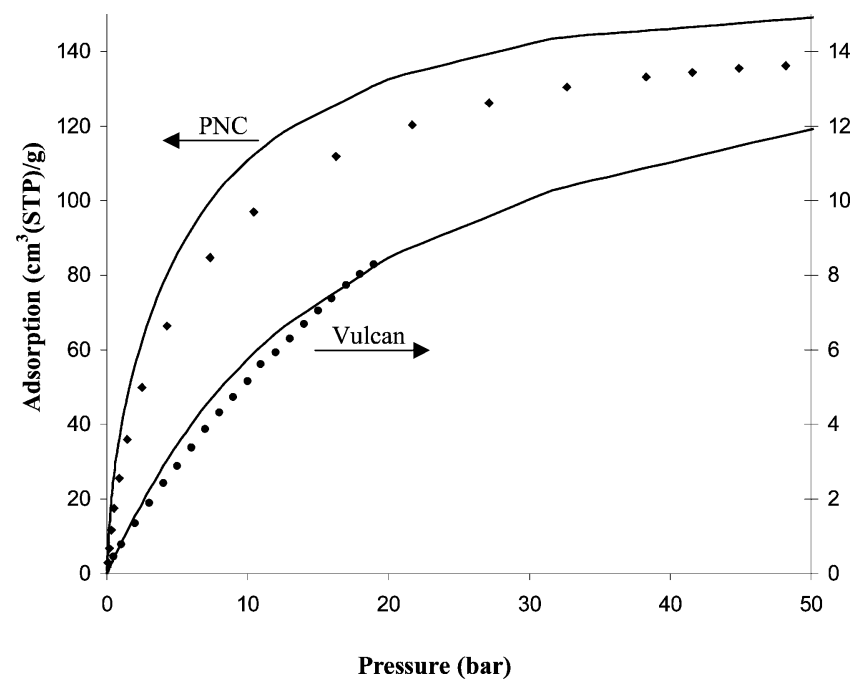

Figure 5. Adsorption isotherms of methane on Vulcan 3G (circles, right axis) and PNC (diamonds, left axis) carbons. The lines are predictions based on a carbon dioxide slit-PSD (see Figure 3) and Monte Carlo simulation kernels with gas-surface interaction strength parameters calibrated to Vulcan 3G. Symbols are experiment.

that calibration of gas-surface interaction parameters to a reference active carbon will be more accurate than calibration to a low surface area carbon for analysis of the properties of active carbons.

Isosteric Heat and the Slab-DFT Model. In part II of this work we use a "coarse-grained" slab-DFT ${ }^{23}$ to predict gas mixture adsorption in active carbons on the basis of pure component gas adsorption isotherms only. A principle feature of this slab-DFT approach is its efficiency. Considering the numerical expense involved in generating a kernel for each pure gas at each temperature for eq 1 in the previous section using MC simulation, we are interested here in assessing the accuracy of the slab-DFT for making adsorption predictions at temperatures other than the temperature at which the slab-DFT is parametrized. In other words, we are interested in the ability of the slab-DFT to convert a kernel at one temperature to another temperature.

The slab-DFT is fully described in previous work. ${ }^{1,23,39}$ Only its main features are described here. As with all DFT approaches applied to materials characterization that we know of, the slabDFT treats gas molecules as hard spheres with a mean-field perturbation describing attractive interactions. Specifically, we approximate the intrinsic excess Helmholtz free-energy with

$$
F^{\mathrm{ex}}=\int \mathrm{d} \mathbf{r} \varphi_{\mathrm{HS}}^{\mathrm{PY}}(\rho\{\mathbf{r}\})+\frac{1}{2} \sum_{i j} \iint \mathrm{d} \mathbf{r}_{1} \mathrm{~d} \mathbf{r}_{2} \rho_{i}\left(\mathbf{r}_{1}\right) \rho_{j}\left(\mathbf{r}_{2}\right) \phi_{i j}^{p}\left(r_{12}\right)
$$

where $\rho_{i}(\mathbf{r})$ is the (nonuniform) density of component $i$ in the slit pore, $\varphi_{\mathrm{HS}}^{\mathrm{PY}}$ is the excess Percus-Yevick compressibility equation of state for a uniform mixture of hard spheres with diameter set $\{d\}$, and $\phi_{i j}^{p}$ is the attractive contribution to the Lennard-Jones potential, defined according to the WCA convention, ${ }^{40,41}$ between particles $i$ and $j$. Our model is presented schematically in Figure 1 of reference 23. It symmetrically parametrizes density profiles in terms of the set $\left\{H_{\mathrm{p}}, \delta H, \rho_{1 i}\right.$, $\left.\rho_{2 i}, \rho_{3 i}, \sigma_{b i}, \delta \sigma_{b i}, z^{*}\right\}$, where each element is nonnegative. Parameters with a subscript ' $i$ ' can be different for each fluid component; otherwise they are the same for all components. The region where the density is zero, described by $\delta H=H_{\mathrm{p}}-$ $H_{\mathrm{c}}$, prohibits fluid particles from overlapping wall particles. The three regions or "slabs" of density represent (1) a monolayer of fluid strongly adsorbed at the wall with density $\rho_{1 i}$ and width $z_{1 i}$, (2) a thick layer of adsorbed fluid with density $\rho_{2 i}$, and (3) the remaining fluid in the center of the slit with density $\rho_{3 i}$ and width $z^{*}$.

For both the external potential and ideal gas contributions of slab 1 to the grand potential, we transform slab 1 so that it has width $\delta \sigma_{b i}$ while conserving the total number of particles, i.e., it has density $\rho_{1 i}{ }^{*}=\rho_{1 i} z_{1 i} / \delta \sigma_{b i}$. Because of this transformation of slab 1 for the external potential and ideal gas contributions only, in effect our prescription for $F^{\mathrm{ex}}$ is a crude nonlocal approximation. In summary, the grand potential is written ${ }^{23}$

$$
\Omega=F^{\mathrm{ex}}+2 A \sum_{i}\left\{\begin{array}{l}
\delta \sigma_{i b} \rho_{1 i}^{*}\left(V_{i}^{\mathrm{ext}}+\ln \left(\Lambda_{i}^{3} \rho_{1 i}^{*}\right)-\mu_{i b}-1\right) \\
+z_{2 i} \rho_{2 i}\left(\ln \left(\Lambda_{i}^{3} \rho_{2 i}\right)-\mu_{i b}-1\right) \\
+z^{*} \rho_{3 i}\left(\ln \left(\Lambda_{i}^{3} \rho_{3 i}\right)-\mu_{i b}-1\right)
\end{array}\right\}
$$

where $A$ is the area of the slit-pore, $z_{2 i}=H_{\mathrm{c}} / 2-z_{1 i}-z^{*}, V_{i}^{\text {ext }}$ is the strength of gas-surface interactions, $\Lambda_{i}^{3}$ is the thermal de Broglie wavelength (an irrelevant constant here), and $\mu_{i b}$ is the chemical potential.

Minimization of eq 8 with respect to the density, with all other parameters held fixed, i.e., variation of $\left\{\rho_{1 i}, \rho_{2 i}, \rho_{3 i}, z^{*}\right\}$ at fixed $\left\{T, H_{\mathrm{p}}, \delta H, \sigma_{b i}, \delta \sigma_{b i}, \sigma_{a i}, \epsilon_{a i}, V_{i}^{\mathrm{ext}}, \mu_{b i}\right\}$, gives the equilibrium state according to this slab model. The bulk LJ parameter set, $\left\{\sigma_{b i}, \epsilon_{b i}\right\}$, is determined by fitting to pure bulk fluid reference pressure-density isotherms for a given temperature, $T$, with $H_{\mathrm{p}} \rightarrow \infty$. We set $d_{i}=\sigma_{a i}$, where $\sigma_{a i}$ is the effective size of an adsorbed particle of type $i$. We also define $\epsilon_{a i}$, the effective interaction energy of an adsorbed particle of type $i$. So $\sigma_{a i}$ and $\epsilon_{a i}$ are determined separately for each slit width, $H_{\mathrm{p}}$. The adsorbed parameter set is determined by estimating $\delta H$ and $\delta \sigma_{b i}$, fixing $V_{i}^{\text {ext }}$ by fitting to the low-density limit of each pure fluid adsorption isotherm, and then fitting $\left\{\sigma_{a i}, \epsilon_{a i}\right\}$ to the entire range of each pure fluid adsorption isotherm. A downhill simplex optimization algorithm ${ }^{28}$ initialized near to $\left\{\sigma_{b i}, \epsilon_{b i}\right\}$ is used to find the local minimum of the rmsd (root-mean-squaredeviation) of this fit.

For prediction of gas adsorption at temperatures other than the temperature at which the DFT is parametrized, the same DFT model is solved to determine $\left\{\rho_{1 i}, \rho_{2 i}, \rho_{3 i}, z^{*}\right\}$ with all the other parameters fixed at the new temperature. This generates the appropriate kernel, which when inserted in eq 1 gives the adsorption isotherm.

For example, Figure 6a shows predictions compared with experiment for adsorption of carbon dioxide at a range of suband supercritical temperatures in CEA active carbon. Note that the PSD for these isotherms is based on the $293 \mathrm{~K}$ carbon dioxide isotherm and the $293 \mathrm{~K}$ slab-DFT kernel. Figure $6 \mathrm{~b}$ shows similar results for PTA8. We see that carbon dioxide adsorption is very well predicted at a range of temperatures, including a temperature $17 \mathrm{~K}$ less than the probe gas temperature. Nitrogen adsorption is also well predicted, methane less so, and the predicted temperature variation of hydrogen is relatively poor. The calculated PSDs for these carbons are shown in Figure 7.

Because Figures $6 \mathrm{a}$ and $6 \mathrm{~b}$ show the temperature variation of adsorption, they can be used together with the isosteric method $^{1}$ to predict the isosteric heat, which in turn can be related to experimental measurements of adsorption heats. The quality of agreement in Figures $6 \mathrm{a}$ and $6 \mathrm{~b}$ demonstrates that adsorption heats, as well as adsorption itself, can be successfully predicted using the models and methods in this work, at least for carbon 

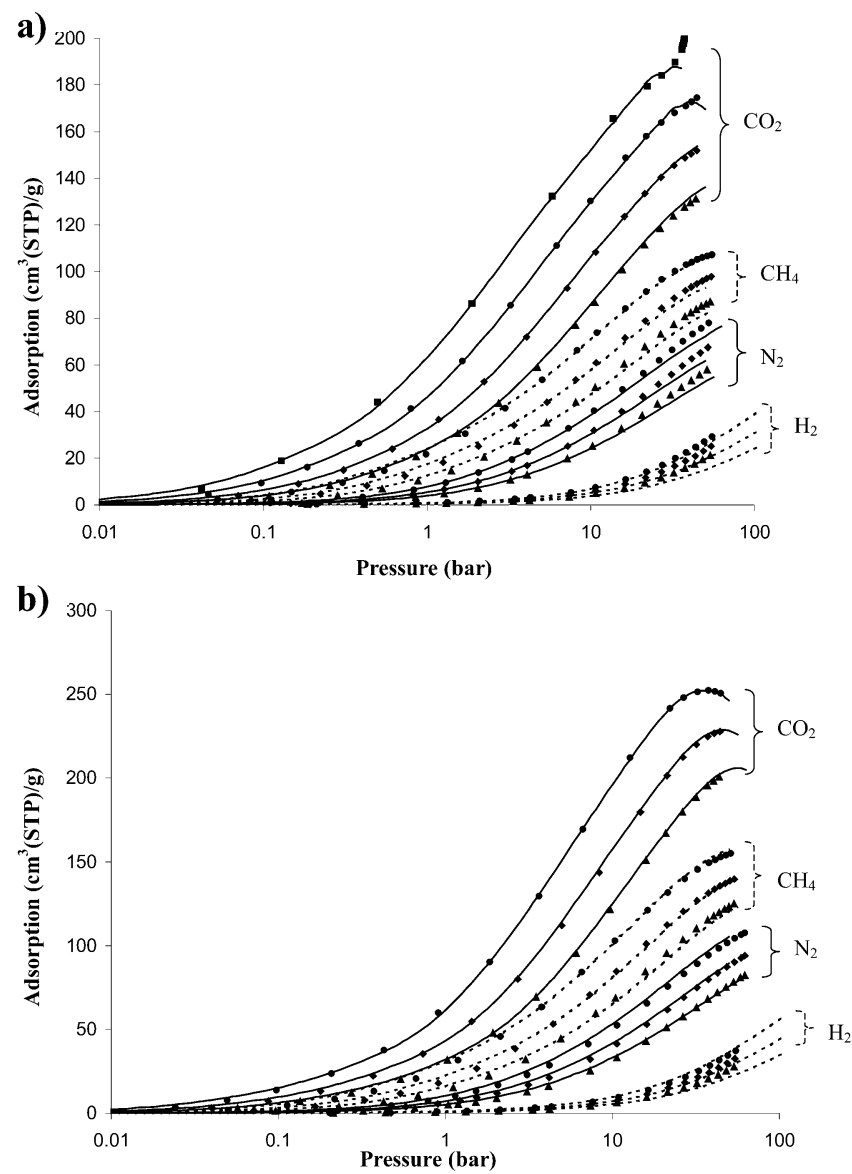

Figure 6. Adsorption isotherms on active carbons: (a) CEA and (b) PTA8. Lines are predictions except for the $293 \mathrm{~K}$ carbon dioxide line, which is fitted to experiment, and symbols are experiment (squares$276 \mathrm{~K}$, circles $-293 \mathrm{~K}$, diamonds $-313 \mathrm{~K}$, triangles-333 K). The predicted isotherms are based on $293 \mathrm{~K}$ carbon dioxide slit-PSDs (see Figure 7) and slab-DFT kernels for each gas at each temperature. Gassurface interaction strength parameters are calibrated to PNC carbon (see Figure 1). The same gas-surface interaction strength is used at each temperature. Pressure is on a logarithmic scale.

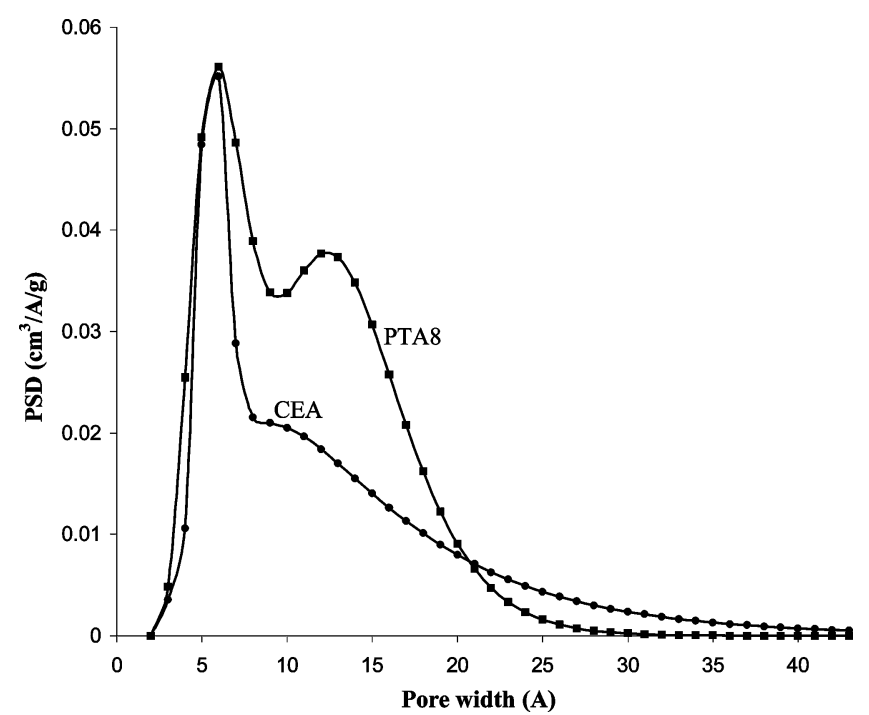

Figure 7. Pore-size distributions (PSDs) for the carbons in Figure 6; CEA (circles) and TA1 (squares).

dioxide and, to a slightly worse degree, nitrogen. Unfortunately the methane, and particularly the hydrogen, predictions overestimate the temperature variation of adsorption and hence the adsorption heat (we do not have experimental adsorption heat data, and so do not make any direct comparisons). But this should not be seen as a failure of the slab-DFT model. Rather, these results indicate that we can fine-tune the $\delta \sigma_{b i}$ parameter for each gas in the slab-DFT (which is currently set on a "onesize-fits-all" basis for all the gases) to match temperature variation or adsorption heat data. Specifically, increasing $\delta \sigma_{b i}$ will result in a smaller value of $V_{i}^{\text {ext }}$ for each pore width, which in turn will lead to a smaller Boltzmann factor. This should have the desired effect of reducing the temperature variation of adsorption and hence reducing adsorption heat prediction.

\section{Discussion}

The results in Figures 2 and 6 are the most accurate results in the literature to date. They demonstrate that the polydisperse independent ideal slit-pore model is a useful model of the surface of active carbons and is accurate for prediction of gas adsorption isotherms and adsorption heats, at least for the relatively simple gases here under the temperature and pressure ranges studied. Note also that our methods remain accurate up to reasonably high pressure, i.e., at least 50 bar, which is significantly higher than most other work in this area. The significant difference between this work and previous work concerns calibration of gas-surface interactions, i.e., we calibrate these interactions to a high surface area carbon, rather than a low surface area carbon. We expect this calibration method to implicitly account for higher-body and weak electrostatic interactions, as well as other complications including the inconsistencies caused by experimental helium calibration of the pore volume, the transferability of our molecular models from a bulk to pore environment, and other heterogeneities not captured by the ideal slit-pore model. Despite this success, we are cautious in recommending these models and methods for use with more complex systems, for example the gas phase adsorption of heavy alkanes or water. It is not yet known whether these models and methods are accurate for molecules with highly nonspherical shapes or with significant electrostatic interactions.

Despite the undoubted success of the polydisperse independent pore model for mimicking the behavior of the systems in this work, it is known to be inadequate for a range of other important systems. Its failure is the result of two factors: (a) the modeled uniformity of individual pores, and (b) the independence of these pores. In real materials there will be both geometric and energetic nonuniformities that the ideal pore model cannot capture. Indeed, it cannot even clearly distinguish energetic from geometric nonuniformity or one kind of geometry from another. ${ }^{42-44}$ Maddox and co-workers ${ }^{45}$ have investigated the influence of pore-junctions on adsorption of a model of nitrogen at $77 \mathrm{~K}$ using molecular simulation. They found that this geometric nonuniformity resulted in pore blocking due to freezing and that similarly sized independent pores did not exhibit this phenomenon. Papadopoulous et al., ${ }^{46}$ Kozak et al., ${ }^{47}$ and $\mathrm{Gelb}^{48}$ have studied the influence of boundary conditions on adsorption in smooth-walled pores of finite length using Monte Carlo simulation and mean-field DFT. Essentially, they find that ideal pores of infinite length exhibit enhanced hysteresis because the formation of menisci is suppressed. For strongly dipolar adsorbates, such as water, Brennan and colleagues ${ }^{49,50}$ find that energetic nonuniformities caused by embedded surface dipoles can potentially lead to pore blocking. And Kierlik and colleagues $^{51-53}$ have demonstrated that pore-pore interactions can significantly affect hysteresis.

Clearly, characterization of nanoporous materials in terms of a polydisperse independent pore model is a gross approximation. For activated carbons high-resolution electron micrographs ${ }^{21,22}$ 
indicate that the slit-pore model might be a reasonable starting point. But there can be value in developing surface models that address specific issues such as energetic or geometric nonuniformity or pore-pore interactions. However, a surface model that can capture all of these effects is likely to be very complex. If energetic and geometric nonuniformity are predominantly short-range in nature, and if pore-pore interactions are longrange, then an accurate model will probably need to describe a wide range of length scales. Essentially, an accurate model will need to capture nanostructure and network topology. Recent articles ${ }^{1,50,54}$ have addressed the development of detailed 3-D surface models in some depth.

\section{Appendix}

In Monte Carlo simulations where model parameters are fitted to reproduce reference data, such as coexisting densities, pairpotentials between molecules without charges or dipoles (i.e., without long-range interactions) can be truncated at a specified cutoff radius, $r_{\mathrm{c}}$, that is less than the minimum simulation box length. Molecules separated by more than this cutoff experience no interaction. This has the advantage that long-range corrections that account for interactions at distances greater than the cutoff and simulation box length are not required. However, in general cutting an analytic potential in this way leaves an undesirable step discontinuity in the interaction. In previous work ${ }^{14}$ we generated optimized gas molecular models for Monte Carlo simulation of gas adsorption in slit pores. However, in that work electrostatic quadrupole interactions between molecules were truncated beyond a particular cutoff radius (between molecular centers), and the effect of this truncation was ignored when calculating pressures on the basis that it probably has little influence. In this work we calculate a new set of optimized molecular models where the effect of potential truncation is properly included. Overall, we find that using these improved molecular models leads to small gains in the accuracy of our methods. This appendix details our newly optimized molecular models and our methods for obtaining them.

A general method for smoothing this discontinuity to facilitate calculation of quantities derived from the gradient of the pair potential, such as pressure, involves applying a ramp for molecule-molecule separations slightly less than $r_{\mathrm{c}}$. So we model the intermolecular potential between two rigid molecules whose centers are separated by a distance $r_{i j}$ by

$$
\phi_{i j}\left(r_{i j}\right)=\left\{\begin{array}{l}
\sum_{a b} \phi_{a b}\left(r_{a b}\right) ; r_{i j}<r_{r} \\
\frac{r_{c}-r_{i j}}{r_{c}-r_{r}} \sum_{a b} \phi_{a b}\left(r_{a b}\right) ; r_{r} \leq r_{i j} \leq r_{c} \\
0 ; r_{i j}>r_{c}
\end{array}\right.
$$

where $\phi$ is a pair potential, $i$ and $j$ are molecular indices, the $a b$ sum is over all pairs of sites ( $a$ sites are on molecule $i$ and $b$ sites are on molecule $j$ ), $\phi_{a b}$ is the "uncut" pair-potential between sites $a$ and $b, r_{a b}$ is the distance between sites $a$ and $b$, and $r_{r}$ $<r_{c}$ is the ramp separation. The bulk pressure calculated via the virial route 55,56 is now

$$
P=k_{\mathrm{B}} T\left\langle\frac{N}{V}\right\rangle-\left\langle\frac{1}{3 V} \sum_{i<j} r_{i j} \frac{\partial \phi_{i j}}{\partial r_{i j}}\right\rangle
$$

where $N$ is the total number of molecules present, $V$ is the

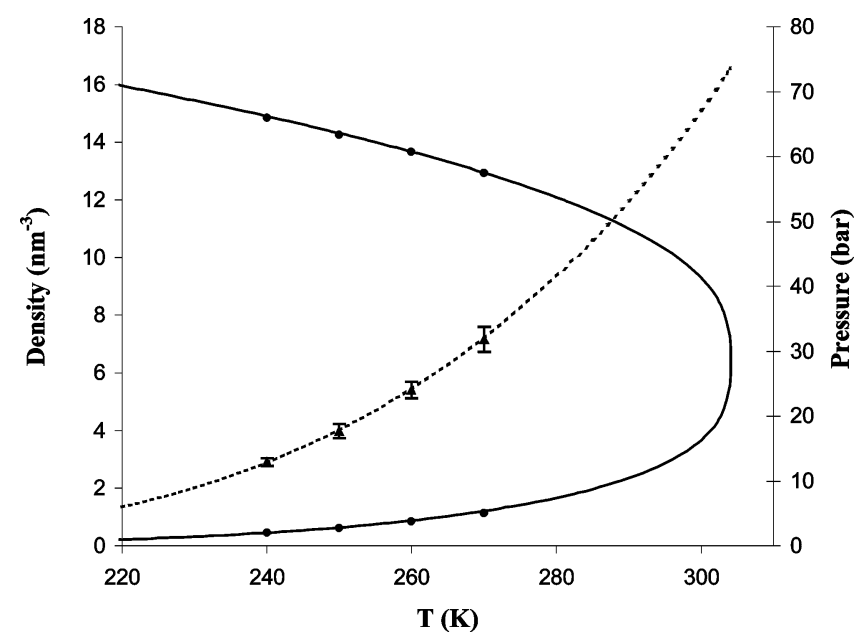

Figure 8. Saturation properties for carbon dioxide from Gibbs ensemble Monte Carlo simulations using the model in Table 1 (symbols) and reference data ${ }^{58}$ (lines). The full lines with circles are coexisting densities while the dotted line with triangles are coexisting pressures.

simulation box volume, and

$$
r_{i j} \frac{\partial \phi_{i j}}{\partial r_{i j}}=\left\{\begin{array}{l}
\sum_{a b} \frac{\partial \phi_{a b}}{\partial r_{a b}} \frac{\mathbf{r}_{a b} \mathbf{r}_{i j}}{r_{a b}} ; r_{i j} \leq r_{r} \\
\sum_{a b} \frac{\partial \phi_{a b}}{\partial r_{a b}} \frac{\mathbf{r}_{a b} \mathbf{r}_{i j}}{r_{a b}}\left(\frac{r_{c}-r_{i j}}{r_{c}-r_{r}}\right)-\frac{r_{i j} \phi_{a b}}{r_{c}-r_{r}} ; r_{\mathrm{r}}<r_{\mathrm{ij}}<r_{\mathrm{c}} \\
r_{\mathrm{ij}}>r_{\mathrm{c}}
\end{array}\right.
$$

where $r_{a b}$ is the length of vector $\mathbf{r}_{a b}$. Neither long-range corrections or discontinuity corrections are now required.We model molecules as rigid assemblies of Lennard-Jones and partial charge sites and set $r_{r}=0.9 r_{c}$. The above truncation regime applies to both Lennard-Jones and electrostatic pair interactions between sites, and we ignore long-range corrections beyond $r_{c}$ (our molecular models are either nonpolar or quadrupolar and so specialized techniques ${ }^{57}$ for handling longrange electrostatic interactions are not needed).

To optimize the gas models for carbon dioxide, methane, and nitrogen we adjust the Lennard-Jones (LJ) parameters until good agreement with experimental data ${ }^{58}$ for saturation properties (coexisting gas and liquid densities and pressures) is obtained by Gibbs ensemble simulation. For hydrogen model optimization we adjust the LJ parameters until a good fit to the bulk fluid isotherm at $293 \mathrm{~K}$ is obtained. We also adjust the nitrogen LJ size parameter in this way, starting from the model obtained by fitting to saturation properties, to improve its molecular model considering that its critical point is about $126 \mathrm{~K}$ while we are interested in adsorption near $293 \mathrm{~K}$. The methane model does not require adjustment in this way. We then use these molecular models, which have been optimized for their performance in bulk simulations, directly in our simulations of gas adsorption in slit pores, ignoring any possible influence of the surface on these models.

Our Gibbs simulations consist of 532 molecules, and a total of 50 million moves are attempted. The first 5 million attempted moves are ignored with respect to calculating statistics. Each grand canonical simulation consists of 5 million attempted moves, with the first 1 million ignored for the purpose of equilibration, and generally consists of several hundred molecules. Statistical errors are calculated in the usual way ${ }^{55}$ based on block averages. We are careful to avoid simulating too close to the critical temperature. 


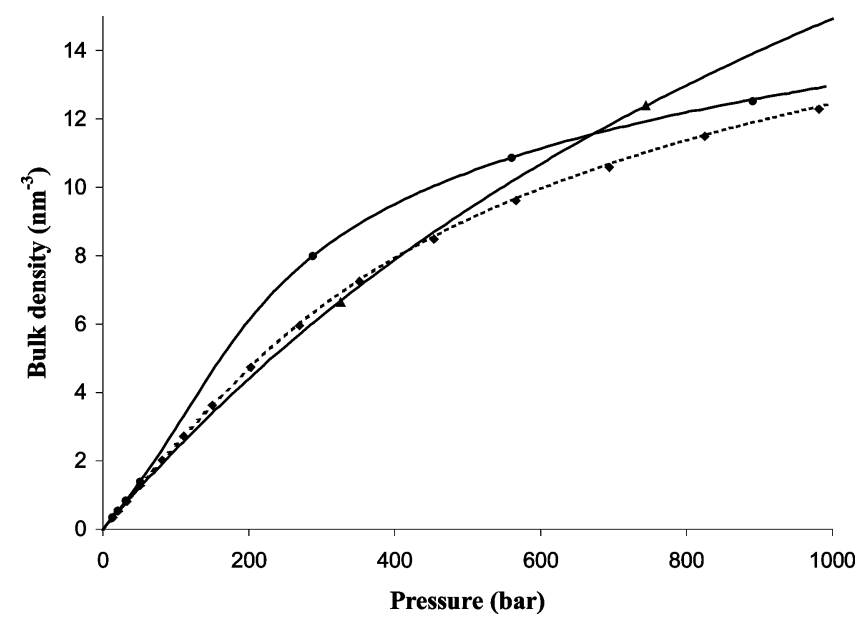

Figure 9. Supercritical bulk isotherms for methane (full line and circles), nitrogen (dashed line and diamonds), and hydrogen (gray line and triangles) from grand canonical ensemble Monte Carlo simulations using the models in Table 1 (symbols) and reference data ${ }^{58}$ (lines).

The resulting molecular models are presented in Table 1. The Gibbs ensemble simulation results for the carbon dioxide model optimized to reproduce saturation properties are shown in Figure 8. Grand canonical ensemble simulation results for bulk supercritical isotherms at $293 \mathrm{~K}$ for the final methane, nitrogen, and hydrogen models are shown in Figure 9.

\section{References and Notes}

(1) Sweatman, M. B.; Quirke, N. Modelling gas adsorption in amorphous nanoporous adsorbents. In Handbook of Theoretical and Computational Nanotechnology; Schommers, W., Rieth, M., Eds.; American Scientific Publishers, in press. 853.

(2) Seaton, N. A.; Walton, J. P. R. B.; Quirke, N. Carbon 1989, 27,

(3) Lastoskie, M. L.; Gubbins, K. E.; Quirke, N. J. Phys. Chem. 1993, 97, 4786.

(4) Ryu, Z. Y.; Zheng, J. T.; Wang, M. H.; Zhang, B. J. J. Colloid Interface Sci. 2000, 230, 312.

(5) Kowalczyk, P.; Terzyk, A. P.; Gauden, P. A.; Leboda, R.; Szmechtig-Gauden, E.; Rychlicki, G.; Ryu, Z. Y.; Rong, H. Q. Carbon 2003, 41, 1113.

(6) Puziy, A. M.; Poddubnaya, O. I.; Martinez-Alonso, A.; SuarezGarcia, F.; Tascon, J. M. D. Appl. Surf. Sci. 2002, 200, 196. 313.

(7) Scaife, S.; Kluson, P.; Quirke, N. J. Phys. Chem. B 2000, 104,

(8) Dombrowski, R. J.; Hyduke, D. R.; Lastoskie, M. L. Langmuir 2000, 16, 5041

(9) Quirke, N.; Tennison, S. R. R. Carbon 1996, 34, 1281.

(10) Gusev, V.; O'Brien, J. A.; Seaton, N. A. Langmuir 1997, 13, 2815.

(11) Lopez-Ramon, M. V.; Jagiello, J.; Bandosz, T. J.; Seaton, N. A. Langmuir 1997, 13, 4435.

(12) Samios, S.; Stubos, A. K.; Kanellopoulos, N. K.; Cracknell, R. F.; Papadopoulos, G. K.; Nicholson, D. Langmuir 1997, 13, 2795.

(13) Samios, S.; Papadopoulos, G. K.; Steriotis, T.; Stubos, A. K. Mol. Sim. 2001, 27, 441 .

(14) Sweatman, M. B.; Quirke, N. J. Phys. Chem. B 2000, 105, 1403.

(15) Sweatman, M. B.; Quirke, N. Langmuir 2001, 17, 5011.

(16) Ravikovitch, P. I.; Vishnyakov, A.; Russo, R.; Neimark, A. V. Langmuir 2000, 16, 2311

(17) Davies, G. M.; Seaton, N. A. AICHE 2000, 46, 1753.

(18) Heuchel, M.; Davies, G. M.; Buss, E.; Seaton, N. A. Langmuir 1999, 15,8695
(19) Gusev, V.; O'Brien, J. A. Langmuir 1998, 14, 6328.

(20) Scaife, S. The Characterization of Porous Carbons Using Computer Simulation and Experimental Techniques; Ph. D. thesis; University of Wales, Bangor, 1999. 381.

21) Harris, P. J. F.; Burian, A.; Duber, S. Philos. Mag. Lett. 2000, 80

(22) Harris, P. J. F. Int. Mater. Rev. 1997, 42, 206.

(23) Sweatman, M. B.; Quirke, N. Langmuir 2002, 18, 10443. 5406.

(25) Gauden, P. A.; Kowalczyk, P.; Terzyk, A. P. Langmuir 2003, 19 , 4253

(26) Kowalczyk, P.; Terzyk, A. P.; Gauden, P. A. J. Colloid Interface Sci. 2001, 243, 300 .

(27) Kowalczyk, P.; Terzyk, A. P.; Gauden, P. A.; Gun'ko, V. M.; Solarz, L. J. Colloid Interface Sci. 2002, 256, 378.

(28) Press, W. H.; Teukolsky, S. A.; Vetterling, W. T.; Flannery, B. P. Numerical recipes in Fortran 77: The Art of Scientific Computing, 2nd ed.; Cambridge University Press: Cambridge, 1992.

(29) Davies, G. M.; Seaton, N. A.; Vassiliadis, V. S. Langmuir 1999 15,8235 .

(30) Rouquerol, F.; Rouquerol, J.; Sing, K. Adsorption by Powders and Porous Solids; Academic Press: New York, 1999.

(31) Steele, W. A. Surf. Sci. 1973, 36, 317.

(32) Steele, W. A. The Interaction of Gases with Solid Surfaces; Pergamon: Oxford, 1974.

(33) Sweatman, M. B.; Quirke, N. Mol. Sim. 2001, 27, 295.

(34) Neimark, A. V.; Ravikovitch, P. I. Langmuir 1997, 13, 5148

(35) Myers, A. L.; Monson, P. A. Langmuir 2002, 18, 10261.

(36) Ozdemir, E.; Morsi, B. I.; Schroeder, K. Langmuir 2003, 19, 9764.

(37) Kluson, P.; Scaife, S. J. Porous Mater. 2002, 9, 115. 2693.

(39) Sweatman, M. B.; Quirke, N. Mol. Sim., in press

(40) Weeks, J. D.; Chandler, D.; Andersen, H. C. J. Chem. Phys. 1971, 54,5237 and 5422

(41) Hansen, J. P.; McDonald, I. R. Theories of Simple Liquids, 2nd ed.; Academic: London, 1986.

(42) Davies, G. M.; Seaton, N. A. Carbon 1998, 36, 1473.

(43) Bojan, M. J.; Steele, W. A. Carbon 1998, 36, 1417.

(44) Gac, W.; Patrykiejew, A.; Sokolowski, S. Thin Solid Films 1997, 298,22

(45) Maddox, M. W.; Quirke, N.; Gubbins, K. E. Mol. Sim. 1997, 19 , 267.

(46) Papadopoulou, A ; van Swol, F.; Marconi, U. M. B. J. Chem. Phys. 1992, 97, 6942

(47) Kozak, E.; Chmiel, G.; Patrykiejew, A.; Sokolowski, S. Phys. Lett. A 1994, 189, 94

(48) Gelb, L. D. Mol. Phys. 2002, 100, 2049

(49) Brennan, J. K.; Thomson, K. T.; Gubbins, K. E. Langmuir 2002 18,5438 .

(50) Brennan, J. K.; Bandosz, T. J.; Thomson, K. T.; Gubbins, K. E. Colloids Surf. A 2001, 187, 539 .

(51) Kierlik, E.; Monson, P. A.; Rosinberg, M. L.; Tarjus, G. J. Phys. Condens. Matter 2002, 14, 9295.

(52) Kierlik, E.; Monson, P. A.; Rosinberg, M. L.; Sarkisov, L.; Tarjus, G. Phys. Rev. Lett. 2001, 87, 055701.

(53) Kierlik, E.; Rosinberg, M. L.; Tarjus, G.; Viot, P. Phys. Chem. Chem. Phys. 2001, 3, 1201 .

(54) Bandosz, T. J.; Biggs, M. J.; Gubbins, K. E.; Hattori, Y.; Iiyama, T.; Kaneko, K.; Pikunic, J.; Thomson, K. T. Molecular Models of Porous Carbons. In Chemistry and Physics of Carbon; Radovic, L. R., Ed.; Marcel Dekker: New York, 2003; Vol. 28.

(55) Allen, M. P.; Tildesley, D. J. Computer Simulation of Liquids; Clarendon Press: Oxford, 1987.

(56) Smit, B.; Frenkel, D. Understanding molecular simulation: from algorithms to applications; Academic: New York, 1996.

(57) Jorge, M.; Seaton, N. A. Mol. Phys. 2002, 100, 2017.

(58) Thermophysical properties of fluid systems. In NIST Chemistry WebBook, 2003 